\title{
MicroRNA-384 regulates both amyloid precursor protein and $\beta$-secretase expression and is a potential biomarker for Alzheimer's disease
}

\author{
CHEN-GENG LIU, JIN-LING WANG, LEI LI and PEI-CHANG WANG \\ Clinical Laboratory of Xuanwu Hospital, Capital Medical University, Beijing 100053, P.R. China
}

Received January 5, 2014; Accepted May 8, 2014

DOI: $10.3892 /$ ijmm.2014.1780

\begin{abstract}
Amyloid precursor protein (APP) and $\beta$-site APP cleaving enzyme (BACE-1) play important roles in the pathogenesis of Alzheimer's disease (AD). In this study, using bioinformatics analysis, we demonstrate that miR-384 is a microRNA (miRNA or miR) predicted to potentially target the 3' untranslated regions (3'-UTRs) of both APP and BACE-1. SH-SY5Y cells were transfected with miR-384 mimic oligonucleotide, miR-384 inhibitor oligonucleotide, or a non-specific control siRNA. We found that the overexpression of miR-384 suppressed the mRNA and protein expression of both APP and BACE-1. The miR-384 inhibitor oligonucleotide induced the upregulation of APP and BACE-1. The activity of BACE-1 was altered following the change in its protein expression. The binding sites of miR-384 on the 3'-UTRs of APP and BACE-1 were identified by luciferase assay. Furthermore, cells were treasted with amyloid- $\beta$ (A $\beta) 42$. A $\beta 42$ downregulated miR-384 expression, leading to the continuous reduction in miR-384 expression. In addition, using a mouse model of AD, as well as patients with mild cognitive impairment (MCI) and dementia of Alzheimer's type (DAT), we examined the levels of miR-384 in cerebral spinal fluid (CSF) and serum. Patients with MCI and DAT had lower blood miR-384 levels compared with the controls. In addition, patients with DAT had lower blood miR-384 levels in blood compared with the MCI group. We also found decreased miR-384 expression in the several cerebral spinal fluid (CSF) of the patients with DAT. Negative correlations were observed between miR-384 and A $\beta 42$ in the serum and CSF from patients with AD. In conclusion, these findings demonstrate that miR-384 may plays a role in the development of $\mathrm{AD}$ and may be a potential non-invasive biomarker for the diagnosis of AD.
\end{abstract}

Correspondence to: Professor Pei-Chang Wang, Clinical Laboratory of Xuanwu Hospital, Capital Medical University, 45 Changchun Road, Beijing 100053, P.R. China

E-mail: peichangwang@yahoo.com.cn

Key words: Alzheimer's disease, amyloid precursor protein, $\beta$-site amyloid precursor protein cleaving enzyme, microRNA

\section{Introduction}

Alzheimer's disease (AD) is a prominent neurodegenerative disorder characterized by the progressive loss of memory and other cognitive functions. Despite considerable progress in genetics and cell biology, there are still a number of unresolved issues regarding the mechanisms responsible for neurodegeneration, as well as the molecular and pathological components. Extracellular amyloid- $\beta(\mathrm{A} \beta)$, which is derived from a larger protein known as amyloid precursor protein (APP), is believed to be responsible for the death of neurons and dementia in AD. The increased APP expression may increase the risk of developing AD $(1,2)$. APP levels can be regulated at the genomic, transcriptional or translational level and participate in the degradation of neurons. Genetic variants in the APP promoter increase APP transcription by 2 to 3 -fold and have been reported to increase the risk of developing AD. APP can be processed by a group of secretases, where $\alpha$-secretase produces soluble fragments, and $\beta$ - and $\gamma$-secretase generate A $\beta$ from APP (2). Several lines of evidence have suggested that $A \beta$ regulates neuronal and synaptic activities and that the accumulation of $\mathrm{A} \beta$ in the brain causes an intriguing combination of aberrant network activity and synaptic depression (3). The $\beta$-secretase, $\beta$-site APP cleaving enzyme (BACE- 1 ), is a ratelimiting enzyme of $A \beta$ generation and a key target of drugs for AD $(1,4,5)$.

MicroRNAs (miRNAs or miRs) are endogenous, short, non-coding RNAs, which act as important post-transcriptional regulators of gene expression by binding with their target mRNAs and are essential for neuronal function and survival $(5,6)$. Several miRs have been shown to be important in neuropathology by downregulating AD-related proteins, such as APP and BACE-1. It has been demonstrated that miR-16, $-101,-106 \mathrm{a} / \mathrm{b},-147$ and -160a function as APP suppressors; another study using an APP/presenilin 1 (PS1) mutant mouse model of AD revealed a negative correlation between BACE-1 protein levels and 2 miRs (miR-298 and -328); miR-298 and -328 downregulated BACE-1 expression through direct interaction (5-7).

Several cerebral spinal fluid (CSF)- or blood-based markers, such as A $\beta$, soluble APP (sAPP) $\alpha /$ sAPP $\beta$, tau, phosphorylated tau (p-tau) and BACE-1, have been proposed as biomarkers for predicting future cognitive decline in healthy 
individuals and the progression to dementia in patients who are cognitively impaired $(8,9)$. However, there is still an urgent need for the availability of more biomarkers that can detect AD in the predementia phase $(1,6,8,9)$. The expression of AD-related proteins, such as APP and BACE-1, is controlled by a number of regulatory factors. The levels of these regulatory factors may be altered before changes occur in the levels of AD-related proteins. Thus, regulatory factors, such as miRs, may serve as potential biomarkers for $\mathrm{AD}$, particulary in the diagnosis of mild cognitive impairment (MCI). In the present study, miRs that potentially target the 3' untranslated region (3'-UTR) of APP or BACE-1 were predicted using retrieve engines, as previously described (10). Bioinformatics analysis revealed that miR-384 was a miR that can potentially target both the 3'-UTRs of APP and BACE-1. The effects of miR-384 on APP and BACE-1 expression were examined, and the levels of miR-384 in samples from mice with $\mathrm{AD}$, as well as in patients with MCI and dementia of Alzheimer's type (DAT) were measured.

\section{Materials and methods}

Study population. This study was approved by the Ethics Committee of Xuanwu Hospital of Capital Medical University, Beijing, China and the written informed consent was obtained from all participants. Thirty-two patients with MCI (13 females, 19 males; mean age, 63.2 \pm 6.1 years), 45 patients with DAT (18 females, 27 males; mean age, 64.2 \pm 5.8 years) were included in this study. A total of 50 control subjects, including 28 females and 22 males were also included (mean age, 63.9 \pm 5.7 years). Matched CSF and blood samples were drawn from 7 patients with DAT ( 2 females, 5 males; mean age, $65.1 \pm 3.5$ years) and 7 control subjects ( 2 females, 5 males; mean age, $66.2 \pm 4.1$ years). Venous blood was collected using a vacuum tube within $2 \mathrm{~h}$ after CSF collection. Serum was isolated within $1 \mathrm{~h}$ after blood collection. The samples were stored at $-80^{\circ} \mathrm{C}$ until analysis. Homocysteine (HCY) and apolipoprotein $\mathrm{E}$ (ApoE) levels were determined using the Hitachi 7600 biochemical analyzer (Hitachi, Ltd., Tokyo, Japan). The $A \beta$, tau and p-tau levels were determined using an ELISA kit (Cusabio Biotech Co., Ltd., Suffolk, UK).

APP/PS1 double-transgenic and wild-type (WT) mice. For our study purposes, 3-, 6- and 9-month-old APP/PS1 doubletransgenic mice with a $\mathrm{C} 57 \mathrm{BL} / 6 \mathrm{~J}$ genetic background were purchased from the Institute of Laboratory Animal Science, Chinese Academy of Medical Sciences and Comparative Medical Center, Beijing, China. All the animal protocols were approved by the Ethics Committee of Xuanwu Hospital of Capital Medical University. Non-transgenic mice were used as the WT controls. The mice were anesthetized with ether and blood was taken by removing the eyeballs, and then CSF-like fluid was collected as previously described (11). Briefly, the mice were sacrificed and their brains were removed into a 35-mm dish. The cranial cavity and cerebral ventricles (lateral, third and fourth ventricles) were rinsed with $1 \mathrm{ml}$ PBS, and CSF was thus harvested with PBS, the washing solution being CSF-like fluid. The hippocampi were then isolated for miR-384 qPCR detection. The samples were placed in liquid nitrogen until analysis. There were 5 mice examined in each group.
Cell culture. The SH-SY5Y and HEK293 cell lines were purchased from the Shanghai Institute of Cell Biology, Shanghai, China. The cells were grown in antibiotic-free DMEM supplemented with $10 \% \mathrm{FBS}$ at $37^{\circ} \mathrm{C}$ with $5 \% \mathrm{CO}_{2}$. Primary mouse hippocampal neurons were isolated from mouse fetuses. Sixteen-day pregnant WT mice were sacrificed by $\mathrm{CO}_{2}$ inhalation, and the primary mouse hippocampal neurons were then isolated as previously described (12).

Transfection. The cells were transfected with $100 \mathrm{nM}$ (final concentration) miR-384 mimic oligonucleotide, miR-384 inhibitor oligonucleotide or a non-specific control siRNA (GenePharma, Shanghai, China) using Lipofectamine ${ }^{\mathrm{TM}} 2000$ reagent (Invitrogen Life Technologies, Carsbad, CA, USA) following the manufacturer's instructions. Cells treated with Lipofectamine 2000 reagent only served as the negative control There were 5 samples tested in each group.

A $\beta 42$ treatment. Synthetic A $\beta 42$ peptides (Bachem, Heidelberg, Germany) dissolved in PBS were aged by incubation at $37^{\circ} \mathrm{C}$ for $24 \mathrm{~h}$ with shaking at $1,000 \mathrm{rpm}$ to allow fibril formation, as previously described (13). The cells were treated for $0,12,24$, 36,48 and $72 \mathrm{~h}$ with either a mock treatment containing PBS or $5 \mu \mathrm{M}$ aged $\mathrm{A} \beta 42$, followed by miR-384 detection. There were 5 samples tested in each group.

5-aza-deoxycytidine (5-Aza-dC) treatment. The SH-SY5Y cells were exposed to $0.2 \mu \mathrm{M} 5$-Aza-dC (Sigma, St. Louis, MO, USA) dissolved in dimethyl sulfoxide for $96 \mathrm{~h}$. The medium and the 5-Aza-dC were replaced every day, as previously described (14). The levels of miR-384 in the harvested cells were detected by qPCR as follows: The $0 \mu \mathrm{M} 5-\mathrm{Aza}-\mathrm{dC}$ group served as the control. The $\mathrm{CpG}$ islands upstream of miR-384 were analyzed by the $\mathrm{CpG}$ Island Searcher. There were 5 samples tested in each group.

Isolation of mRNA and $q P C R$ analysis. Total RNA from the harvested cells was isolated using TRIzol Reagent (Invitrogen Life Technologies). The isolated RNA was reverse transcribed using PrimeScript ${ }^{\mathrm{TM}}$ RT reagent (Takara Bio, Inc., Shiga, Japan). The mRNA expression of APP and BACE-1 was determined using SYBR ${ }^{\circledR}$-Green qPCR (Takara Bio, Inc.) in the Light Cycler 480 System (Roche Diagnostics GmbH, Mannheim, Germany). Glyceraldehyde-3-phosphate dehydrogenase (GAPDH) was used to normalize the target genes. The primers used for PCR were as follows: APP forward, 5'-TTGCGAAACTCATC TTCACTGG-3' and reverse, 5'-CAGTGGGCAACACACAAA CTCTAC-3'; BACE-1 forward, 5'-AGGCAGTCTCTGGTAT ACACCCATC-3' and reverse, 5'-TGCCACTGTCCACAATG CTC-3'; and GAPDH forward, 5'-GCACCGTCAAGGCTGAG AAC-3' and reverse, 5'-TGGTGAAGACG CCAGTGGA-3'.

Isolation of miRs and $q P C R$ analysis. Total RNA from the CSF, plasma and serum samples was extracted by a spin column method using the miRNeasy Serum/Plasma kit (Qiagen, Hilden, Germany) according to the manufacturer's instructions. Total RNA in the hippocampal tissues from the animals and cultured cells was extracted by a spin column method using the miRNeasy kit (Qiagen). The miRs were reverse transcribed into cDNA using the miScript II RT kit 


\section{6: 5'-uauauuugugauaUAGGAAu-3' APP | I | | | | \\ 3'-auaCUUGUUAAAGAUCCUUa-5' hsa-miR-384

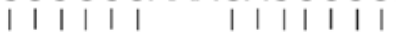 623: 5'-uggGAUCAA-agCUAGGAAa-3' BACE-1}

Figure 1. The seed regions of miR-384 in the 3'-UTRs of amyloid precursor protein (APP) and $\beta$-site APP cleaving enzyme (BACE-1).

(Qiagen) in 10- $\mu 1$ reaction system. miR-384 was detected by the TaqMan qPCR method (Qiagen), using U6 snRNA as an endogenous control.

Reporter vectors and DNA constructs. Reporter vectors containing the putative miRNA target sites from the APP and BACE-1 3'-UTRs were synthesized with double-stranded oligonucleotides perfectly complementary to putative miRNA target sites and oligonucleotides in which the seed regions were mutated. The APP oligonucleotides had following the sequence (seed region shown in bold): 5'-CCCAAGCTTTATATTTGT GATATAGGAATAAGCTTGGG-3' and 3'-GGGTTCGAAA TATAAACACTATATCCTTATTCGAACCC-5'. The mutant APP target oligonucleotides had nucleotides 3 through 6 of the seed region mutated (italicized): 5'-CCCAAGCTTTATATTT GTGATATCGTAGTAAGCTTGGG-3' and 3'-GGGTTCG AAATATAAACACTATAGCATCATTCGAACCC-5'. The BACE-1 oligonucleotides had the sequence (seed region shown in bold): 5'-CCC AAGCTTTGGGATCAAAGCTAGGAAA AAGCTTGGG- 3' and 3'-GGGTTCGAAACCCTAGTTTC GATCCTTTTTCGAACCC-5'. The mutant BACE-1 target oligonucleotides had nucleotides 7 through 13 of the seed region mutated (italicized): 5'-CCCAAGCTTTGGTACCCACGCC ATGCGAAAGCTTGGG-3' and 3'-GGGTTCGAAACCAT GGGTGCGGTACGCTTTCGAACCC-5'. We utilized established methods to clone these synthetic versions of the putative miRNA target sites into a luciferase reporter gene (pMIR-REPORT; Ambion Inc., Austin, TX, USA) (11). A total of 10,000 HEK-293 cells were plated in 24 well plates. The following day, the cells were transfected with a miRNA mimic oligonucleotide, reporter vectors bearing either the miRNA target sequence or the miRNA seed region mutant target sequence, and one tenth of the molar volume of pRL-SV40, a Renilla luciferase control vector. We utilized Arrest-In transfection reagent (Open Biosystems Inc., Lafayette, CO, USA); any differences in transfection efficiency were accounted for by measuring Renilla luciferase activity. At $48 \mathrm{~h}$ post-transfection, the cells were lysed using $100 \mu 1$ of GLB (Glo Lysis Buffer; Promega, Madison, WI, USA). Firefly and Renilla luciferase activities were measured using a dual luciferase reporter assay kit (Promega), as per the manufacturer's instructions. Firefly luciferase activity was normalized to Renilla luciferase activity. There were 5 samples examined in each group.

Western blot analysis. Western blot analysis was performed as previously described (15). Briefly, proteins (30 $\mu \mathrm{g} /$ well) were separated by $10 \%$ SDS-polyacrylamide gel electrophoresis (SDS-PAGE; Bio-Rad, Hercules, CA, USA). Proteins in the gel were transferred to nitrocellulose membranes (Pall Life Sciences, Port Washington, NY, USA). In the sequence, the membranes were incubated with anti-APP antibody (diluted 1:400; Abcam, London, UK), anti-BACE-1 antibody (diluted 1:1,000, Abcam), or anti-GAPDH antibody (diluted 1:400, Abcam) at room temperature for $1.5 \mathrm{~h}$. The membranes were washed and incubated with anti-IgG antibody conjugated to horseradish peroxidase at room temperature for $1 \mathrm{~h}$. Subsequently, the membranes were incubated with substrate for peroxidase and chemiluminescence enhancer (KPL Inc., Gaithersburg, MD, USA) for $1 \mathrm{~min}$ and exposed immediately to X-ray film for 1-5 min. The films were then revealed in the conventional manner. The amount of each protein was measured by densitometric analysis and standard relative to the GAPDH. There were 5 samples examined in each group.

In vitro BACE-1 activity assay. BACE-1 activity was determined by a fluorescence resonance energy transfer method using a kit from Sigma. The BACE-1 activity in [fluorescence units (FU)] was quantified through the standard curve. Negative control (no enzyme) reaction and standard curve blank were contained in the assay. There were 5 samples examined in each group. All reactions were performed in duplicate.

Statistical analysis and ROC curve analysis. Statistical analyses were performed using SPSS 13.0 software for Windows. For normally distributed data, the results are expressed as the means \pm standard deviation (SD); differences between 2 groups were assessed by t-tests. Differences between multiple groups were analyzed using the Mann-Whitney U test, while correlations were determined by computing Spearman rank correlation coefficients. P-values $<0.05$ were considered to indicate significantly different differences.

\section{Results}

Bioinformatics analysis. A total of $62 \mathrm{miRs}$ were found to be putatively target on the 3'-UTR of BACE-1, and 34 miRs were found to be putatively target on the 3'-UTR of APP. miR-384 was an miR that may target the 3 '-UTRs of BACE-1 and APP (Fig. 1).

MiR-384 suppresses the expression of BACE-1 and APP. As illustrated in Figs. 2 and 3A-D, both the mRNA and protein expression of BACE-1 and APP was markedly decreased following transfection with miRNA-384 mimic oligonucleotide in primary mouse hippocampal neurons and SH-SY5Y cells $(\mathrm{P}<0.05)$. The miR-384 inhibitor oligonucleotide induced a significant upregulation in the mRNA and protein expression of APP and BACE-1 compared with the groups transfected with the non-specific control siRNA or the negative control $(\mathrm{P}<0.05)$. We observed a $57 \%$ downregulation in endogenous miR-384 under this condition (Fig. 3E). The activity of BACE-1 in the primary mouse hippocampal neurons and SH-SY5Y cells was also significantly suppressed by miR-384 and was significantly increased by the miR-384 inhibitor oligonucleotide $(\mathrm{P}<0.05)$ (Fig. 3F).

The UTRs of BACE-1 and APP 3' are both targets of miR-384. The overexpression of miR-384 significantly reduced fluorescence from the APP and BACE-1 reporter vectors in the HEK 293 cells $(\mathrm{P}<0.05)$. These reductions were not observed 
A

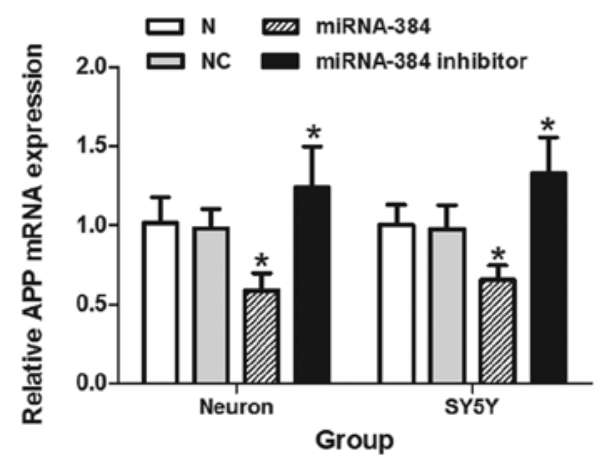

B

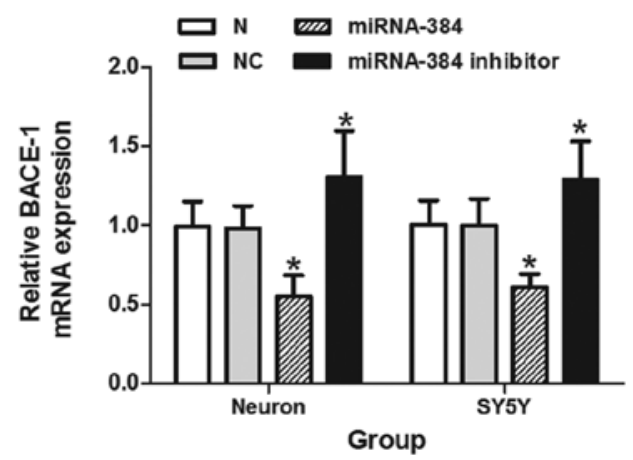

Figure 2. The effects of miR-384 and miR-384 inhibitor on the mRNA expression of (A) amyloid precursor protein (APP) and (B) $\beta$-site APP cleaving enzyme (BACE-1) were examined using SH-SY5Y cells (SY5Y). N, negative controls. NC, non-specific control. " $\mathrm{P}<0.05$ compared with NC group.

A

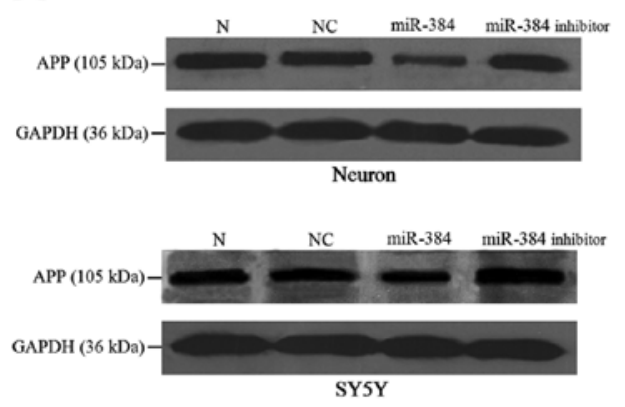

C
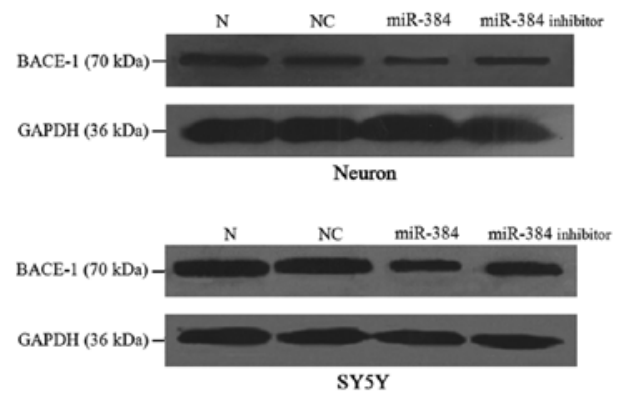

$\mathbf{E}$

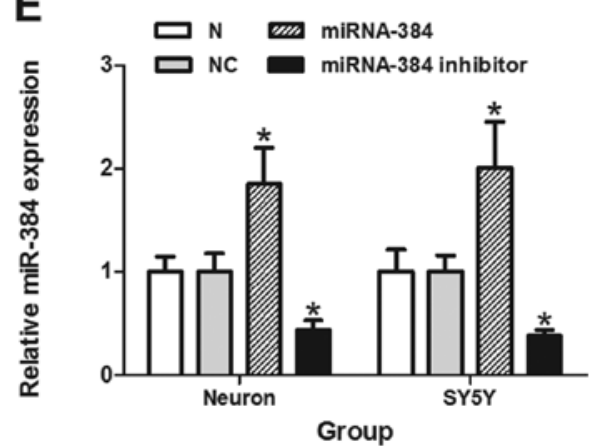

$\mathbf{B}$

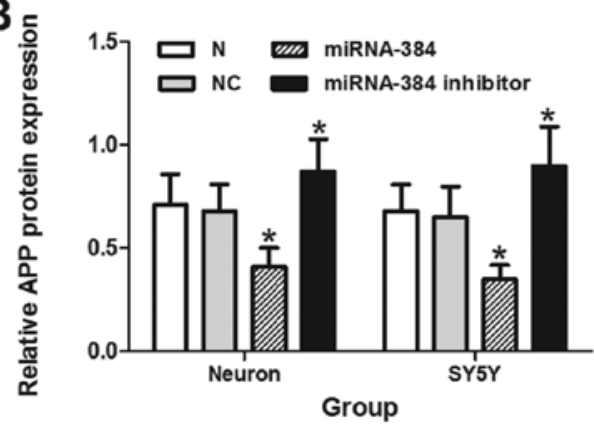

D

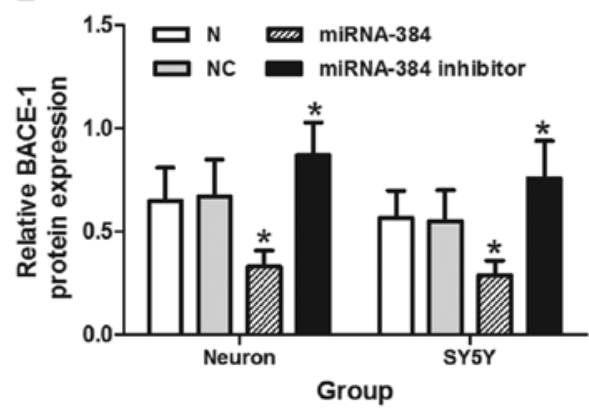

$\mathbf{F}$

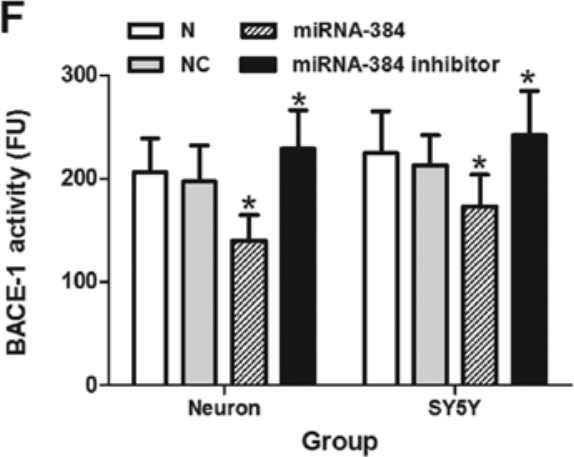

Figure 3. The effects of miR-384 and miR-384 inhibitor on the protein expression of (A and B) amyloid precursor protein (APP), (C and D) $\beta$-site APP cleaving enzyme (BACE-1), (E) intracellular miR-384 levels and (F) activity of BACE-1 were examined using neurons and SH-SY5Y cells (SY5Y). N, negative controls. NC, non-specific control. $* \mathrm{P}<0.05$ compared with $\mathrm{NC}$ group.

when the seed regions mutants of the APP or BACE-1 3'-UTRs were utilized (Fig. 4).
A 342 downregulates miR-384 expression in vitro. 5 -Aza-dC at a concentration of $0.2 \mu \mathrm{M}$ did not affect miR-384 expression 


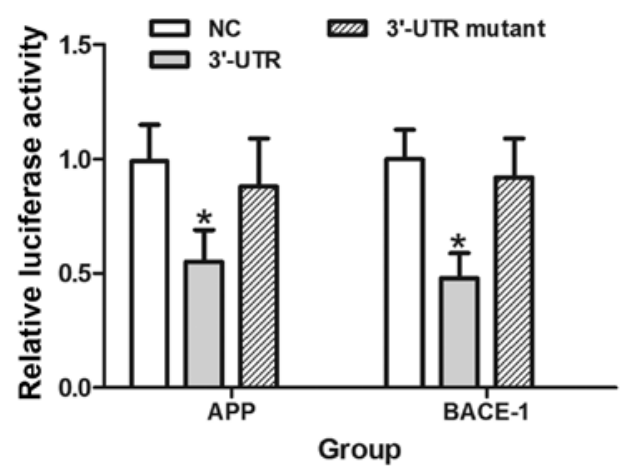

Figure 4. Binding sites of miR-384 on the 3'-UTRs of amyloid precursor protein (APP) and $\beta$-site APP cleaving enzyme (BACE-1) were identified. NC, non-specific control. ${ }^{*} \mathrm{P}<0.05$ compared with the $\mathrm{NC}$ group.

in the SH-SY5Y cells ( $\mathrm{P}>0.05)$ (Fig. 5A). miR-384 expression decreased after $12 \mathrm{~h}$ of incubation with $5 \mu \mathrm{M}$ aged $\mathrm{A} \beta 42$, and continued to decline until $48 \mathrm{~h}$ (Fig. 5B). The results of bioinformatics analysis suggested that there was no $\mathrm{CpG}$ island upstream of miR-384.

Expression of miR-384 is decreased in the hippocampus, CSF-like fluid and serum of transgenic mice. The levels of miR-384 were significantly downregulated in the hippocampi, CSF-like fluid and serum obtained from the 3-, 6- and 9-month-old APP/PS1 transgenic mice compared with the WT mice $(\mathrm{P}<0.05)$. The levels of miR-384 in the hippocampi, CSF-like fluid and serum of the 6- and 9-month-old transgenic mice were significantly lower than those in the hippocampi, CSF-like fluid and serum from the 3-month-old transgenic mice $(\mathrm{P}<0.05)$ (Fig. 6A-C).

Expression of miR-384 is decreased in the CSF, serum and plasma of patients with AD. Compared with the control groups, patients with MCI and DAT had lower levels of miR-384 in the serum and plasma $(\mathrm{P}<0.05)$. The patients with DAT had lower miR-384 levels in their serum and plasma compared with the MCI groups $(\mathrm{P}<0.05)$. We also found decreased a miR-384 expression in the CSF of patients with DAT compared with the control group $(n=7)(P<0.05)$ (Fig. 6D and E).

The levels of miR-384 obtained from plasma or serum strongly correlated with each other $(r=0.957, \mathrm{P}<0.05)$, indicating that both serum and plasma samples are suitable for investigations of miR-384 as blood-based biomarkers (Fig. 6F). The levels of miR-384 were lower in the CSF than those in the serum from a given individual $(\mathrm{P}<0.05)$ (data not shown). There was no correlation observed in miR-384 expression in the CSF and serum from a given individual (data not shown). When the cut-off values were set as 0.771 and 0.526 according to the ROC curve analysis, the positive rates of serum miR-384 were $53.1 \%(17 / 32)$ and $66.7 \%(30 / 45)$ of the MCI and DAT subjects, respectively.

miR-384 negatively correlates with $A \beta 42$ in serum and CSF. A weak but significant negative correlation was observed between the levels of miR-384 and A $\beta 42$ in the serum obtained from patients with $\mathrm{MCI}(\mathrm{r}=-0.372, \mathrm{P}<0.05)$ and DAT $(\mathrm{r}=-0.437$, $\mathrm{P}<0.05)$, as well as the control group $(\mathrm{r}=-0.463, \mathrm{P}<0.05)$. A
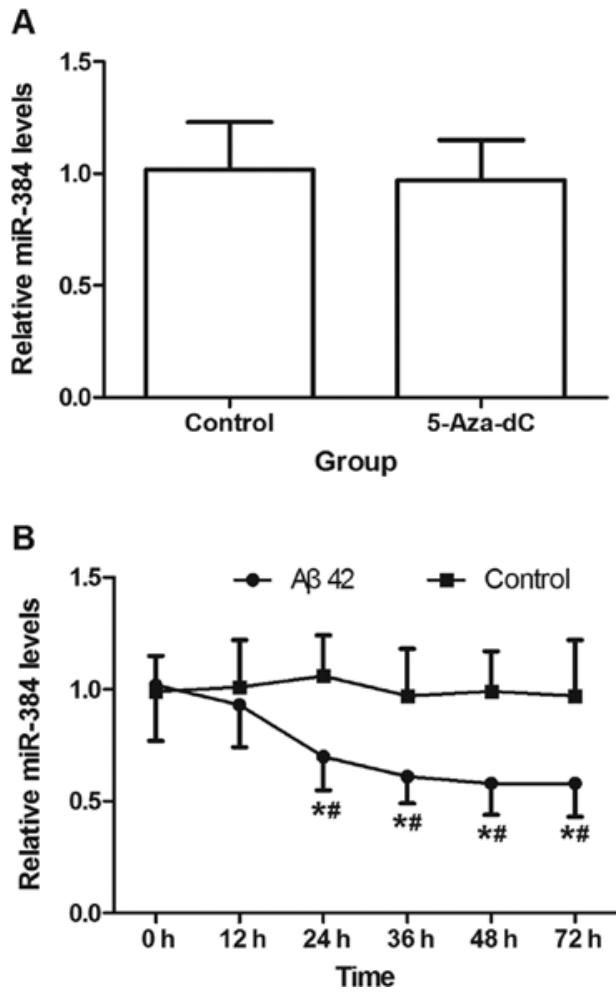

Figure 5. Effects of (A) 5-aza-deoxycytidine (5-Aza-dC) and (B) amyloid- $\beta$ (A $\beta) 42$ on the expression of miR-384 in SH-SY5Y cells. ${ }^{*} \mathrm{P}<0.05$ compared with the control group; ${ }^{\#} \mathrm{P}<0.05$ compared with the 0 - and 12 -h groups (B).

stronger negative correlation was observed between the levels of miR-384 and $A \beta 42$ in the CSF obtained from patients with DAT $(r=-0.571, \mathrm{P}<0.05)$ and the control group $(\mathrm{r}=-0.577$, $\mathrm{P}<0.05)$. miR-384 expression showed no correlation with HCY, ApoE, tau and p-tau (data not shown).

\section{Discussion}

miRs are integral components of biological networks with fundamental roles in regulating gene expression $(1,2,5)$. miR profiles are known to be altered in several regions of the brain in $\mathrm{AD}$; however, the cause or consequence of the disease remains unknown. There are no data suggesting a direct genetic link between miRs or miR recognition elements and neurodegenerative disease (6-8). The increased expression of APP correlates with the accelerated accumulation of $A \beta$ in the brain in AD. As the rate-limiting enzyme of $\mathrm{A} \beta$ generation, $\mathrm{BACE}-1$ is a key drug target for AD. In the present study, we found that miR-384 suppressed the expression of APP and BACE-1 by binding their 3'-UTRs. The activity of BACE-1 was also suppressed by miR-384, which coincided with the the change in its protein expression. The suppressive effects of miR-384 on BACE-1 and APP expression may provide a new direction for targeted therapy for AD. This may allow researchers to suppress two key proteins using one miR, which may prove to be more effective with fewer side-effects.

The increase in A $\beta 42$ expression is caused by the aberrant processing of APP, which is the major constituent of senile plaques in the brain in AD (16). It has been demonstrated that $\mathrm{A} \beta 42$ affects miR profiles (17). miR downregulation in 
A
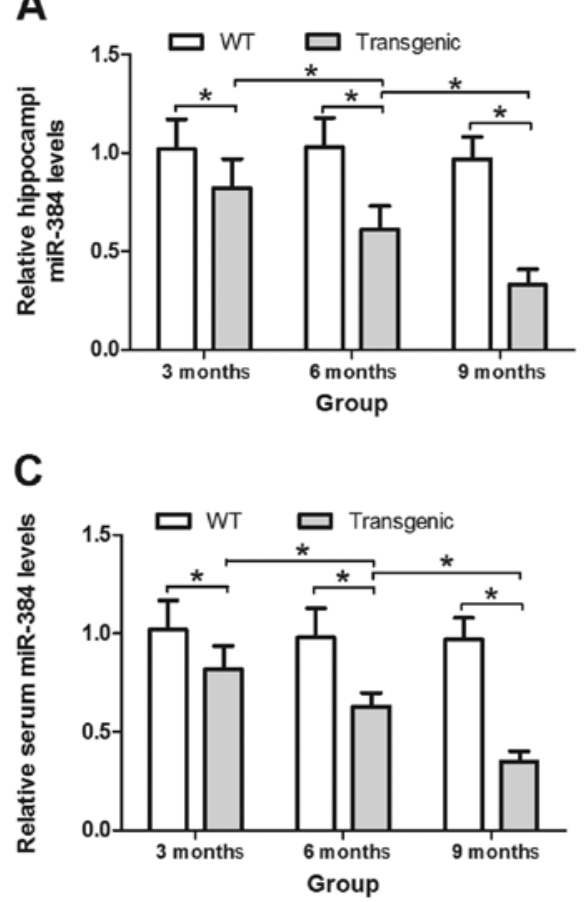

E

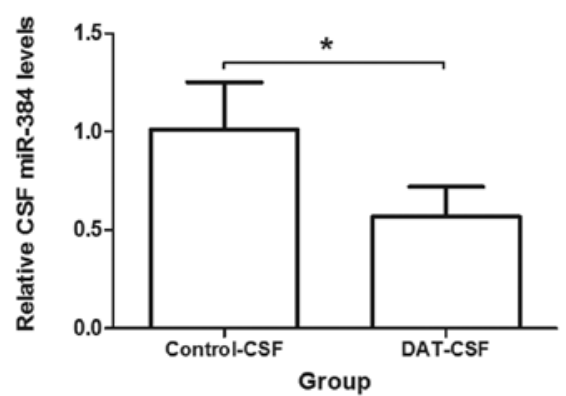

B

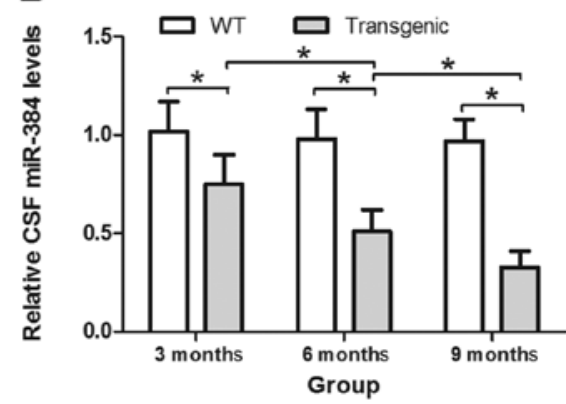

D

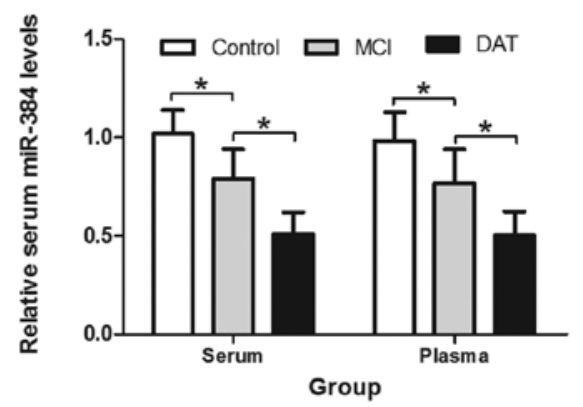

$\mathbf{F}$

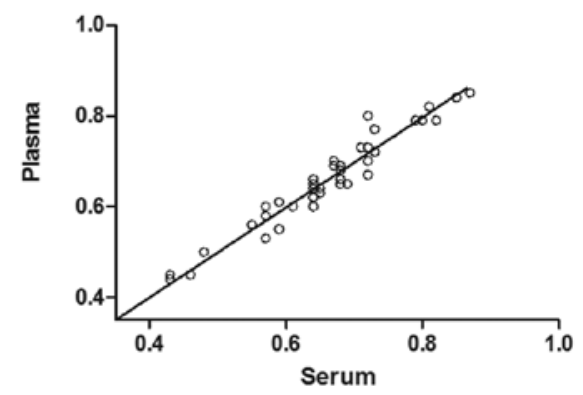

Figure 6. (A-C) The levels of miR-384 in the hippocampus, blood and several cerebral spinal fluid (CSF) of amyloid precursor protein (APP)/presenilin 1 (PS1) transgenic mice (at 3,6 and 9 months of age) and (D and E) patients with mild cognitive impairment (MCI) or dementia of Alzheimer's type (DAT) were determined by TaqMan qPCR. (F) The correlation of miR-384 levels in matched samples of serum or plasma collected from a given individual was analyzed. ${ }^{*} \mathrm{P}<0.05$. WT, wild-type mice.

A $\beta$-treated hippocampal neurons was observed in the hippocampus of $\mathrm{A} \beta$-plaque forming APP23 mice at the onset of plaque formation (13). In this study, the downregulation of miR-384 expression by $\mathrm{A} \beta 42$ in vitro indicates that excessive A 342 generated from transgenic APP may play a role in the downregulation of miR-384 in APP/PS1 double-transgenic mice, and the decreased expression of miR-384 may lead to the upregulation of APP. In this manner, a vicious circle is generated; this vicious circle may play a role in the progression of $\mathrm{AD}$.

For the majority of diseases, multiple biomarkers rather than a particular one are required for diagnosis. CSF is in direct contact with the extracellular space of the brain and can reflect biochemical changes that occur in the latter $(17,18)$. For these reasons, CSF is the optimal source of AD biomarkers. However, CSF is not an appropriate sample for the screening and routine test as requires an invasive process of sample collection. Previous studies have demonstrated that miRNAs are stably expressed in animal serum/plasma, and that their unique expression patterns can serve as 'fingerprints' of various diseases $(18,19)$. Thus, blood-based biomarkers for
AD would be ideal. APP/PS1 double-transgenic mice contain insoluble amyloid peptides at the age of 6-9 months, concomitant with the formation of amyloid plaques (19). In this study, we found that the level of miR-384 was decreased in the hippocampi of 3-month-old transgenic mice, which suggests that the change in miR-384 expression occurs earlier than the formation of amyloid plaques. The detection of miR-384 in the CSF-like fluid and serum of 3-, 6- and 9-month-old transgenic mice demonstrates that miR-384 is a potential AD biomarker, particularly durin the earlier stages. This conjecture was further confirmed by clinical detection, which showed that the miR-384 level in patients with MCI was higher than the control group and lower than that in patients with DAT in the CSF and blood samples. This indicates that the level of miR-384 may change with the progression of $\mathrm{AD}$ and may serve as a biomarker for MCI and DAT.

There is no direct evidence that miRs can move across the blood-brain barrier (BBB) freely. However, some miRs can be selectively packaged into microvesicles, exosomes and actively secreted, and a microvesicle-mediated secretion pathway exists 
in BBB $(18,20)$. Although circulating miRs can be derived from many sources, the decreased secretion of miR-384 may lead to the decreased level of miR-384 in the blood.

In conclusion, these findings demonstrate that miR-384 may play a role in the development of $\mathrm{AD}$, and shows great potential as a novel, non-invasive and easily detected bloodbased biomarker for MCI and DAT.

\section{Acknowledgements}

This study was supported by grants from the Natural Science Foundation of China (no. 81271924) and the Research Fund for the Doctoral Program of Higher Education of China (no. 20121107110001). The authors would like to thank Dr Shuang Meng of the Chinese Center for Disease Control and Prevention, Beijing, China for the vector construction and fluorescence detection.

\section{References}

1. Weiner MW: Dementia in 2012: further insights into Alzheimer disease pathogenesis. Nat Rev Neurol 2: 65-66, 2013.

2. Singer O, Marr RA, Rockenstein E, et al: Targeting BACE1 with siRNAs ameliorates Alzheimer disease neuropathology in a transgenic model. Nat Neurosci 8: 1343-1349, 2005.

3. Tan L, Yu JT, Hu N and Tan L: Non-coding RNAs in Alzheimer's disease. Mol Neurobiol 47: 382-393, 2013.

4. Junn E and Mouradian MM: MicroRNAs in neurodegenerative diseases and their therapeutic potential. Pharmacol Ther 133: 142-150, 2012

5. Schonrock N, Matamales M, Ittner LM and Götz J: MicroRNA networks surrounding APP and amyloid- $\beta$ metabolism - implications for Alzheimer's disease. Exp Neurol 235: 447-454, 2012.

6. Boissonneault V,Plante I, Rivest S and Provost P: MicroRNA-298 and microRNA-328 regulate expression of mouse beta-amyloid precursor protein-converting enzyme 1. J Biol Chem 284: 1971-1981, 2009.
7. Faghihi MA, Modarresi F, Khalil AM, et al: Expression of a noncoding RNA is elevated in Alzheimer's disease and drives rapid feed-forward regulation of beta-secretase. Nat Med 14: 723-730, 2008.

8. Blennow K, Hampel $\mathrm{H}$, Weiner $\mathrm{M}$ and Zetterberg $\mathrm{H}$ : Cerebrospinal fluid and plasma biomarkers in Alzheimer disease. Nat Rev Neurol 6: 131-144, 2010.

9. Zetterberg H, Andreasson U, Hansson O, et al: Elevated cerebrospinal fluid BACE1 activity in incipient Alzheimer disease. Arch Neurol 65: 1102-1107 2008.

10. Schonrock N, Ke YD, Humphreys D, et al: Neuronal microRNA deregulation in response to Alzheimer's disease amyloid-beta. PLoS One 5: e11070, 2010.

11. Patel N, Hoang D, Miller N, et al: MicroRNAs can regulate human APP levels. Mol Neurodegener 3: 10, 2008.

12. Wei PC, Tsai CH, Chiu PS and Lai SC: Matrix metalloproteinase-12 leads to elastin degradation in BALB/c mice with eosinophilic meningitis caused by Angiostrongylus cantonensis. Int J Parasitol 41: 1175-1183, 2011.

13. Schonrock N, Humphreys DT, Preiss T and Götz J: Target gene repression mediated by miRNAs miR-181c and miR-9 both of which are down-regulated by amyloid- $\beta$. J Mol Neurosci 46: 324-335, 2012.

14. Lindner DJ, Wu Y, Haney R, et al: Thrombospondin-1 expression in melanoma is blocked by methylation and targeted reversal by 5-Aza-deoxycytidine suppresses angiogenesis. Matrix Biol 32: 123-132, 2013.

15. Liu CG, Xu KQ, Xu X, et al: 17Beta-oestradiol regulates the expression of $\mathrm{Na}^{+} / \mathrm{K}^{+}$-ATPase beta1-subunit, sarcoplasmic reticulum $\mathrm{Ca}^{2+}$-ATPase and carbonic anhydrase iv in $\mathrm{H} 9 \mathrm{C} 2$ cells. Clin Exp Pharmacol Physiol 34: 998-1004, 2007.

16. Giedraitis V, Sundelöf J, Irizarry MC, et al: The normal equilibrium between CSF and plasma amyloid beta levels is disrupted in Alzheimer's disease. Neurosci Lett 427: 127-131, 2007.

17. Dassow H and Aigner A: MicroRNAs (miRNAs) in colorectal cancer: from aberrant expression towards therapy. Curr Pharm Des 19: 1242-1252, 2013.

18. Zhang Y, Liu D, Chen X, et al: Secreted monocytic miR-150 enhances targeted endothelial cell migration. Mol Cell 39: 133-144, 2010.

19. van Leuven F: Single and multiple transgenic mice as models for Alzheimer's disease. Prog Neurobiol 61: 305-312, 2000.

20. Ma R, Jiang T and Kang X: Circulating microRNAs in cancer: origin, function and application. J Exp Clin Cancer Res 31: 38, 2012 . 